\title{
Neuroendocrine Tumor of Jejunum which (68) Ga-DOTATOC Positron Emission Tomography-Computed Tomography (PET/CT) was Useful
}

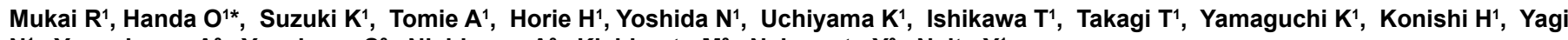
$\mathbf{N}^{1}$, Yanagisawa $\mathrm{A}^{2}$, Yasukawa $\mathrm{S}^{2}$, Nishimura $\mathrm{A}^{2}$, Kishimoto $\mathbf{M}^{2}$, Nakamoto $\mathrm{Y}^{3}$, Naito $\mathbf{Y}^{1}$

${ }^{1}$ Department of Gastroenterological Medicine, Kyoto Prefectural University Graduate School of Medicine, Kyoto City, Kyoto, Japan

${ }^{2}$ Department of Pathology, Kyoto Prefectural University Graduate School of Medicine, Kyoto City, Kyoto, Japan

${ }^{3}$ Department of Diagnostic Imaging and Nuclear Medicine, Kyoto University, Kyoto City, Kyoto, Japan

\begin{abstract}
A 59-year-old man was suspected to have metastatic liver tumors. As the biopsy finding from the liver tumor specimen was consistent with a metastatic neuroendocrine tumor, (68) Ga-DOTATOC positron emission tomography-computed tomography (PET/CT) was performed, and an abnormal uptake was detected in the jejunum. Finally, biopsy of the specimen obtained using double-balloon endoscopy revealed a neuroendocrine tumor in the jejunum. Neuroendocrine tumor of the small intestine is difficult to diagnose before surgery. If a neuroendocrine tumor was strongly suspected, it is recommended to perform a (68) Ga-DOTATOC PET/CT.
\end{abstract}

Keywords: Neuroendocrine tumor; Small intestine; Carcinoid; DOTATOC-PET; Biopsy

\section{Introduction}

Neuroendocrine tumors (NETs; also called carcinoid tumors) are rare, accounting for only approximately $2 \%$ of all gastrointestinal tumors [1]. In Japan, gastrointestinal carcinoid tumors are often found in the hind intestines (the rectum and large intestine) and fore intestines (the stomach, duodenum, and esophagus) but not in the mid intestines (the jejunum, ileum, and appendix) [2]. In 2010, the World Health Organization (WHO) classification of NETs was updated (WHO classification 2010) to include the histopathology and tumor biology of these tumors [3]. WHO classification 2010 was defined based on the number of nuclear mitoses and Ki-67 labeling index. According to this system, NETs were classified as grade 1 (NET-G1) or grade 2 (NET-G2). The so-called carcinoid tumors include NET-G1. NET of the small intestine is difficult to diagnose. One reason is that jejunal carcinoid tumors rarely occur. Moreover, NETs in the small intestine are usually found in solitary form, and only $29-41 \%$ of them occur in the multiple form [1]. Up to $93 \%$ of patients with small intestinal NET are diagnosed at an advanced stage of the carcinoid tumor, with metastases to regional lymph nodes, the liver, or the peritoneum [4]. Patients rarely present clinical symptoms like in the case presented herein. Indeed, patients may have symptoms such as intermittent stomachache, weight loss, fatigue, diarrhea, and vomiting if they have obstruction, ischemia, or bleeding in the small intestine because of primary tumors. If they have metastatic liver tumors and poor detoxification ability for serotonin, they may have skin flush, diarrhea, and endocardial fibrosis, which are characteristic of carcinoid syndrome. The classic carcinoid syndrome is observed only in $20-30 \%$ of patients [5]. The ratio of patients with carcinoid syndrome before surgical resection has been reported to range from $10 \%$ to $15 \%$ [6]. Consequently, these factors make diagnosis in the early stage of the disease difficult, and most of these tumors are discovered incidentally during laparotomy or in the clinical course of the occult primary cancer with hepatic metastases [5]. Similarly, in the present case, hepatic metastases were found at first without any symptoms.

NETs have been reported frequently; however, only a few cases of intestinal NET have been reported. In this report, we describe a case of a jejunal NET which (68) Ga-DOTATOC positron emission tomography - computed tomography (PET/CT) was useful.

\section{Case Presentation}

A 59-year-old man who had been followed up for multiple liver hemangioma at a family medicine clinic for 2 years underwent ultrasonography (US), which revealed the increased size of the liver hemangioma. Consequently, he was introduced to our hospital for further examination. He had a history of premature atrial contraction and had taken oral medication with anti-arrhythmic agent. Physical examination revealed no remarkable signs of any diseases. Blood chemistry findings were almost within the normal limits, and tumor markers did not show abnormal values (alpha-fetoprotein, $3.4 \mathrm{ng} / \mathrm{mL}$; protein induced by vitamin $\mathrm{K}$ absence or antagonist II, $26 \mathrm{mAU} / \mathrm{mL}$; carcinoembryonic antigen, $1.0 \mathrm{ng} / \mathrm{mL}$; and carbohydrate antigen $19-9$, $4.0 \mathrm{U} / \mathrm{mL}$ ). Abdominal US revealed fatty liver and two liver tumors at S3 and S7, which were approximately $1 \mathrm{~cm}$ in diameter and showed a "bull's-eye" appearance, suggesting the possibility of metastatic liver tumors (Figure 1). Abdominal dynamic contrast-enhanced computed tomography (CT) also supported the possibility of metastatic liver tumors because of the ring enhancement of the tumors at S3 and S7 (Figures $2 \mathrm{a}$ and $2 \mathrm{~b}$ ). To search for the primary lesion of the liver tumors, (18) F-FDG positron emission tomography/computed tomography (FDG-PET) was performed. However, no abnormal (18) F-FDG uptake was found in the liver and other parts of the body. In addition, colonoscopy and chest CT could not detect the primary lesion of the liver tumors.

Finally, we performed a biopsy of the liver tumor. Microscopic findings from the biopsy specimen showed that the tumor cells formed glandular and rosette patterns and were composed of nests of uniform cells with small oval-shaped nuclei and an eosinophilic cytoplasm (Figures 3a-3d). Immunohistochemical examination revealed that the

*Corresponding author: Handa $\mathrm{O}$, Department of Gastroenterologica Medicine, Kyoto Prefectural University Graduate School of Medicine, Kyoto City, Kyoto, Japan, Tel: +81-75-251-551; E-mail: handao@koto.kpu-m.ac.jp

Received September 19, 2018; Accepted September 21, 2018; Published September 25, 2018

Citation: Mukai R, Handa O, Suzuki K, Tomie A, Horie H, et al. (2018) Neuroendocrine Tumor of Jejunum which (68) Ga-DOTATOC Positron Emission Tomography-Computed Tomography (PET/CT) was Useful. J Clin Case Rep 8: 1172. doi: 10.4172/2165-7920.10001172

Copyright: @ 2018 Mukai R, et al. This is an open-access article distributed under the terms of the Creative Commons Attribution License, which permits unrestricted use, distribution, and reproduction in any medium, provided the original author and source are credited. 
Citation: Mukai R, Handa O, Suzuki K, Tomie A, Horie H, et al. (2018) Neuroendocrine Tumor of Jejunum which (68) Ga-DOTATOC Positron Emission Tomography-Computed Tomography (PET/CT) was Useful. J Clin Case Rep 8: 1172. doi: 10.4172/2165-7920.10001172

Page 2 of 4

tumor cells were positive for chromogranin, synaptophysin, CD56 (focal), CAM5.2, cytokeratin (CK) 19, and heat shock protein (HSP)70, but negative for CK20, CK7, Hepa, and alpha-fetoprotein. Additional immunohistochemical staining was positive for somatostatin receptor type 2 (SSTR2) and Ki-67. The Ki-67 labeling index was $<1 \%$. Based on these findings, we made a diagnosis of NET-G1 of the liver.

Considering that primary hepatic NETs are extremely rare [7], we strongly suspected that the liver tumors might be metastatic lesions of a NET with an unknown primary lesion. To search for the primary tumor, upper gastrointestinal endoscopy and capsule endoscopy (CE) were performed but did not detect the primary lesion in the whole gastrointestinal tract.

In Europe, an imaging tool with a somatostatin analog has been used to diagnose NETs [8]. We performed (68) Ga-DOTA-d-Phe (1)-Tyr (3)-octreotide (DOTATOC) PET/CT because the tumor cells were positive for SSTR2. As a result, an abnormal (68) Ga-DOTATOC uptake was found in the jejunum and mesenteric lymph nodes (Figure 4). Oral double-balloon endoscopy (DBE) detected a yellowish submucosal tumor covered by normal mucosa with extended small vessels on its surface, in the lower part of the jejunum (Figures 5a-5d). Gastrografin contrast imaging with DBE revealed an "apple-core" sign at the part of the tumor but no abnormalities at the remaining small intestine. The biopsy finding from the specimen obtained from the top of the tumor was consistent with jejunal NET grade 1 (NET-G1), also called carcinoid tumor of the jejunum (Figure 6).

The final diagnosis was NET-G1 of the jejunum with metastasis in the mesenteric lymph nodes and liver. To confirm the number of metastatic liver tumors, we performed dynamic magnetic resonance (MR) imaging with gadolinium-ethoxybenzyl-diethylenetriamine penta-acetic acid (Gd-EOB-DTPA) and found eight tumors that showed early staining in the arterial phase and washout pattern, which suggested the possibility of metastatic liver tumors.

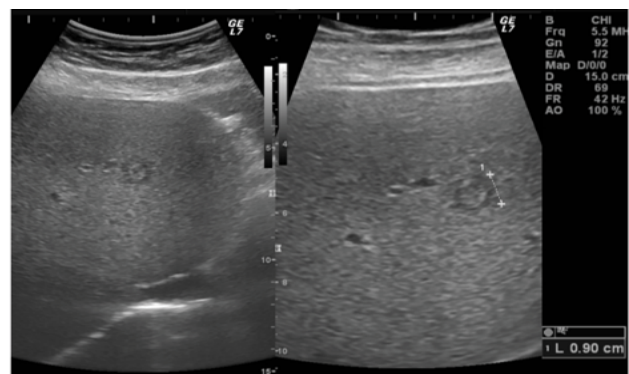

Figure 1: Abdominal Ultra sonography (US) revealed the liver tumor at $\mathrm{S} 7$, which were approximately $1 \mathrm{~cm}$ in diameter and showed a "bull's-eye" appearance.

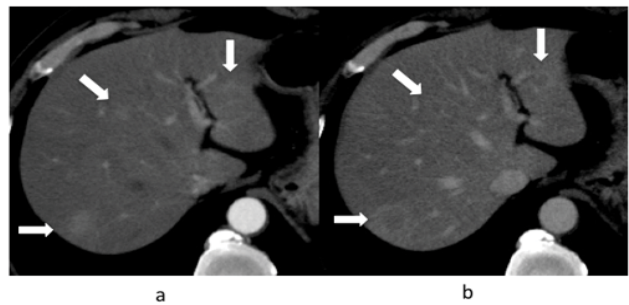

Figure 2: Abdominal dynamic contrast-enhanced computed tomography (CT) showed liver tumors that exhibit ring enhancement (arrows). Arterial dominant phase (2a), and Delayed phase (2b).
After 3 months from his first consultation, partial resection of the small intestine, including the NET, and resection of the multiple metastatic liver tumors and mesenteric lymph nodes were successfully performed. Macroscopic examination revealed that the NET was in the jejunum, $160 \mathrm{~cm}$ from the ileocecal valve (Figures $7 \mathrm{a}$ and $7 \mathrm{~b}$ ), nine nodular brownish tumors were in the liver (S2, S3, and S6-S8), and the mesenteric lymph nodes were swollen. Histological examination

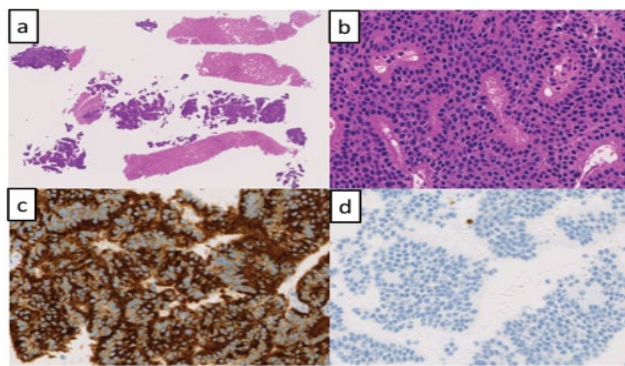

Figure 3: Microscopic findings from the biopsy specimen showed that the tumor cells formed glandular and rosette patterns and were composed of nests of uniform cells with small oval-shaped nuclei and an eosinophilic cytoplasm on $\mathrm{H} \& \mathrm{E}$ staining $(3 \mathrm{a}, 40 \mathrm{x}, 3 \mathrm{~b}, 400 \mathrm{x}$ ) and immunochemical staining for chromogranin A (3c, 400x) and MIB-1 (3d, 200x)
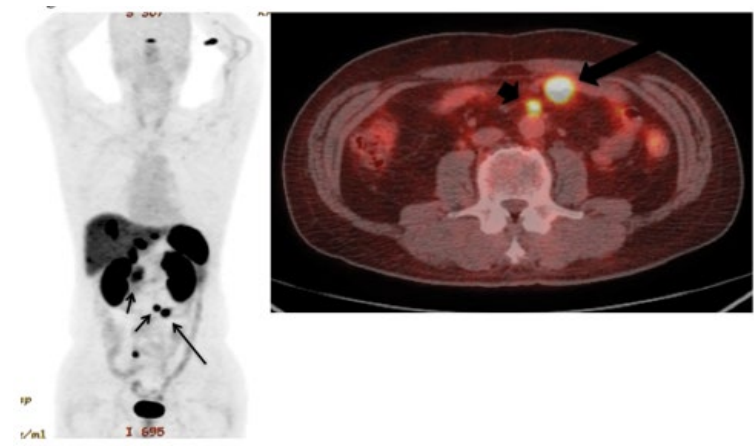

Figure 4: An abnormal (68) Ga-DOTATOC uptake was found in the jejunum (long arrow) and mesenteric lymph nodes (short arrow).

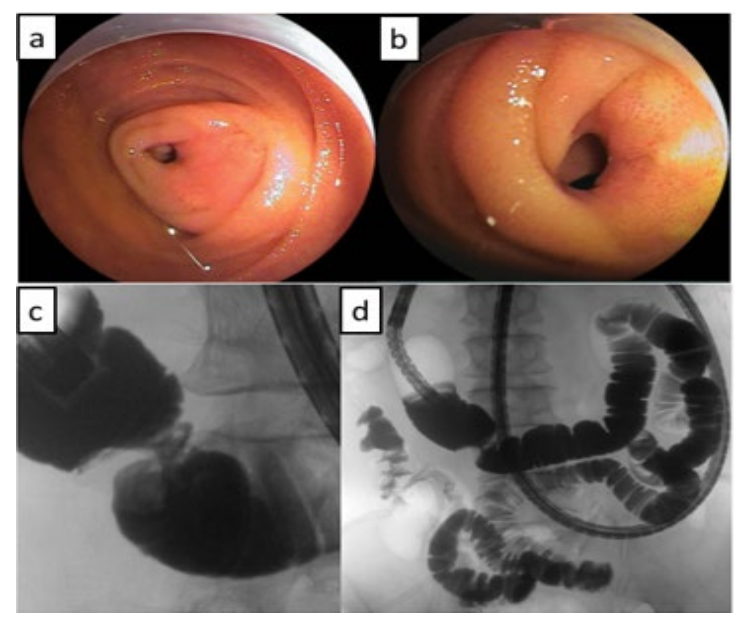

Figure 5: Oral DBE detected a yellowish submucosal tumor covered by normal mucosa with extended small vessels on its surface, in the lower part of the jejunum $(5 a, 5 b)$. Gastrografin contrast imaging with DBE revealed an "apple-core" sign at the part of the tumor $(5 \mathrm{c})$, but no abnormalities at the remaining small intestine $(5 \mathrm{~d})$. 


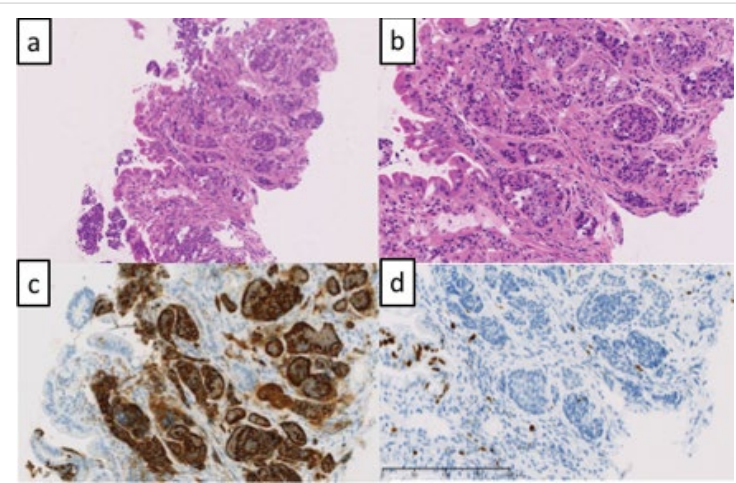

Figure 6: The biopsy from the specimen obtained from the top of the tumo was consistent with jejunal NET grade 1 (NET-G1), also called carcinoid tumor of the jejunum on $\mathrm{H}$ and $\mathrm{E}$ staining (6a. 40x, 6b, 100x) and immunochemical staining for chromogranin A $(6 \mathrm{c}, 100 \mathrm{x})$ and MIB-1 $(6 \mathrm{~d}, 100 \mathrm{x})$

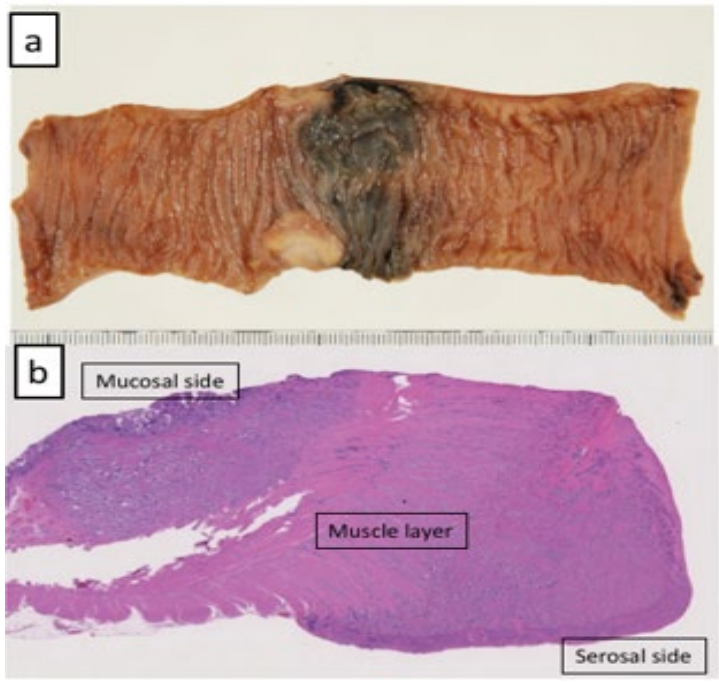

Figure 7: NET was in the jejunum, $160 \mathrm{~cm}$ from the ileocecal valve (7a) Histological examination of the resected specimen revealed a nodular NET deeply invading the submucosal layer and partially exposed to the serosal layer (7b loupe statue)

of the resected specimen revealed a nodular NET deeply invading the submucosal layer and partially exposed to the serosal layer. Synaptophysin and chromogranin A staining revealed positive cells in the jejunal tumor. The possibility of residual tumors in the liver was suggested because the partial resection margin of the metastatic lesions in the liver was histologically positive for NET. Two months later, we performed trans arterial chemoembolization (TACE) with cisplatin (CDDP) and oral administration of octreotide (a long-acting somatostatin analog, i.e., an SSTR2-preferring agonist that also has moderate affinity for SSTR3 and SSTR5). At the latest follow-up, 7 years after the first diagnosis, no recurrence was observed.

\section{Discussion}

NETs of the small intestine are difficult to diagnose before surgery. The diagnostic yield of NET of the small intestine before surgery is low (4.8\%) [9]. However, diagnosis of NET of the small intestine at an early stage is important because of the high rates of positive lymph nodes or liver metastasis as the stage advances. In this case, liver metastasis was detected and diagnosed as NET of the liver, and then a primary lesion in the small intestine was suspected on (68) Ga-DOTATOC PET/CT.
Finally, the jejunal NET was confirmed as the primary lesion by using endoscopic biopsy with DBE.

Considering that the primary hepatic NET is extremely rare [7], we examined the gastrointestinal tract to identify the primary lesion. In addition to the upper and lower gastrointestinal endoscopy, CE is usually performed as a less invasive and acceptable examination of the small intestine. In this case, CE was performed but did not detect the jejunal NET. The diagnosis rate of a single tumor or tumors of $<2 \mathrm{~cm}$ in size is not so high at approximately 37.5\% [10]. Submucosal tumors (SMTs) can be easily overlooked on CE [11]. In this case, the tumor had the shape of an SMT, which was difficult to discover.

In this case, no primary lesion could be found in the gastrointestinal tract by routine endoscopy, and FDG-PET was performed for further examination, but no apparent uptake was detected. The detection rate of NETs by FDG-PET is not necessarily as high as $36 \%$ to $83 \%$ [12] because the proliferative activity of NETs is not as high as that of carcinoma. In this case, the Ki-67 labeling index, the marker of proliferation, in liver biopsy showed low proliferation activity, and the positive staining for the somatostatin receptor (SSTR2) indicated the well-differentiated characteristic of this tumor. As a result, PET-CT detected the primary lesion. Therefore, we performed (68) Ga-DOTATOC PET/CT, which has high affinity for SSTR2, after obtaining written informed consent from the patient. In Japan, this is not approved by the public health insurance in April 2018. As a result, DOTATOC PET/CT detected a primary lesion in the jejunum, and multiple metastasis in the liver and lymph nodes. As $80-100 \%$ well-differentiated NETs express SSTRs [13], somatostatin analog scintigraphy has been used to detect the location of carcinoid tumors. SSTRs are of 5 types, and the imaging examinations with a high affinity with SSTR2 and SSTR5, which are expressed in many gastrointestinal NETs, are single-photon emission computed tomography (SPECT) with indium-111 and PET/CT with (68) Ga-DOTATOC. In Europe, (68) Ga-DOTATOC PET/CT, more sensitive than SPECT, has been used for 10 years; however, in Japan, (68) Ga-DOTATOC-PET/CT can be used only in clinical studies. The approval of (68) Ga-DOTATOC for NET in health insurance is awaited.

Although small intestinal carcinoid is a slowly progressing tumor, the 5-year survival rate of noncurative resection cases of primary lesions is approximately $50 \%$; even in the case of curative resection, the 5-year relapse-free survival period is approximately $80 \%$ [14]. Therefore, early diagnosis and curative resection are required for better prognosis. Regarding liver metastasis, even if curative surgery cannot be performed, appropriate postoperative treatment can improve the life prognosis [15]. In this case, the primary tumor was curatively resected. Although the liver metastatic lesions were not curatively resected because the partial resection margin was histologically positive for NET, TACE and oral administration of somatostatin analogue was performed after surgery. As a result, the patient has survived without relapse for 7 years since the first diagnosis.

\section{Conclusion}

Diagnosis of small intestinal NETs at an early stage and appropriate treatment is important. If a neuroendocrine tumor was strongly suspected, it is recommended to aggressively perform a (68) GaDOTATOC PET/CT. In the future, we expect this diagnostic tool will become widely available for NETs.

\section{References}

1. Horton KM, Kamel I, Hofmann L, Fishman EK (2004) Carcinoid tumors of the small bowel: A multitechnique imaging approach. AJR Am J Roentgenol 182 559-567. 
Citation: Mukai R, Handa O, Suzuki K, Tomie A, Horie H, et al. (2018) Neuroendocrine Tumor of Jejunum which (68) Ga-DOTATOC Positron Emission Tomography-Computed Tomography (PET/CT) was Useful. J Clin Case Rep 8: 1172. doi: 10.4172/2165-7920.10001172

2. Ito $\mathrm{T}$, Igarashi $\mathrm{H}$, Nakamura $\mathrm{K}$ (2015) Epidemiological trends of pancreatic and gastrointestinal neuroendocrine tumors in Japan: A nationwide survey analysis. J Gastroenterol 50: 58-64.

3. Fottner C, Weber MM (2012) Neuroendocrine neoplasms of the gastrointestinal tract. Classification, clinical presentation and diagnosis. Internist (Berl) 53: 131-144.

4. Chambers AJ, Pasieka JL, Dixon E, Rorstad O (2010) Role of imaging in the preoperative staging of small bowel neuroendocrine tumors. J Am Coll Surg 211: $620-627$.

5. Musholt TJ (2011) Extent of resection for neuroendocrine tumors of the smal intestine. Chirurg 82: 591-597.

6. Appetecchia M, Baldelli R (2010) Somatostatin analogues in the treatment of gastroenteropancreatic neuroendocrine tumours, current aspects and new perspectives. J Exp Clin Cancer Res 29: 19.

7. Cerwenka H (2012) Neuroendocrine liver metastases: Contributions of endoscopy and surgery to primary tumor search. World J Gastroenterol 18: 1009-1014.

8. Graham MM, Gu X, Ginader T, Breheny P, Sunderland JJ (2017) (68) GaDOTATOC imaging of neuroendocrine tumors: A systematic review and metaanalysis. J Nucl Med 58: 1452-1458.
9. Morimatsu K, Kuga H, Shinagawa Y (2015) A case of small intestinal carcinoid tumor diagnosed by chance because of a lymph node metastasis Nihon Rinshogeka Gakkai Zasshi. J Japan Surg Ass 76: 789-792.

10. Honda W, Ohmiya N, Hirooka Y (2012) Enteroscopic and radiologic diagnoses, treatment, and prognoses of small-bowel tumors. Gastrointest Endosc 76: 344-354.

11. Shibata H, Hashimoto S, Shimizu K (2015) Beneficial effects of combining computed tomography enteroclysis/enterography with capsule endoscopy for screening tumor lesions in the small intestine. Gastroenterol Res Pract 2: 1-8.

12. Binderup T, Knigge $U$, Loft $A$ (2010) Functional imaging of neuroendocrine tumors: a head-to-head comparison of somatostatin receptor scintigraphy, 123I-MIBG scintigraphy, and 18F-FDG PET. J Nucl Med 51: 704-712.

13. Ito T, Jensen RT (2017) Molecular imaging in neuroendocrine tumors: recent advances, controversies, unresolved issues, and roles in management. Curr Opin Endocrinol Diabetes Obes 24: 15-24.

14. CG M (1989) The clinical presentation and natural history of carcinoid tumours of the gastroin- testinal tract. Stomach Intest 24: 859-868.

15. Coriat R, Walter T, Terris B, Couvelard A, Ruszniewski P (2016) Gastroenteropancreatic Well-Differentiated Grade 3 Neuroendocrine Tumors: Review and Position Statement. Oncologist 21: 1191-1199. 